\title{
Event-related Potential Patterns Reflect Reversed Hemispheric Activity during Visual Attention Processing in Children with Dyslexia: A Preliminary Study
}

\author{
Joong-Gu Kang ${ }^{1,2}$, Seung-Hwan Lee ${ }^{2,3}$, Eun-Jin Park ${ }^{3}$, Hyun-Sung Leem ${ }^{1}$ \\ 'Department of Optometry, Eulji University, Daejeon, ${ }^{2}$ Clinical Emotion and Cognition Research Laboratory, Goyang, ${ }^{3}$ Department of \\ Psychiatry, Inje University IIsan Paik Hospital, Goyang, Korea
}

\begin{abstract}
Objective: Individuals with dyslexia experience reading difficulties, whereas their other cognitive abilities seem normal. The purpose of this study was to investigate the event-related potential (ERP) patterns of children with dyslexia during a target-detection task.

Methods: Seventeen children with dyslexia and 18 children without this disorder participated in this study. We evaluated their writing and reading ability, symptoms of attention-deficit/hyperactivity disorder, and intelligence quotient. ERPs were recorded while participants performed a target-detection task, and the peak amplitude and latency of P100 and P300 were analyzed. The lateral asymmetry index (LAl) was calculated for each ERP component.

Results: The dyslexic group exhibited longer reaction times and larger P100 amplitudes than the non-dyslexic group in the right hemisphere. The P100 latency was also significantly delayed in the right hemisphere of those in the dyslexic group compared with those in the non-dyslexic group. The P300 amplitude was larger in the right hemisphere compared with left hemisphere in the dyslexic group, whereas no interhemispheric differences were observed with respect to the P300 latency. The LAl for P100 showed a significant right hemispheric dominance, whereas the LAl for P100 was significantly correlated with the accuracy of target detection in children with dyslexia.

Conclusion: Our results suggest that right hemispheric dominance acts as an ancillary system that compensates for poor reading in children with dyslexia.
\end{abstract}

KEY WORDS: Dyslexia; Visual attention; Event-related potential; Right hemisphere; Child.

\section{INTRODUCTION}

Despite adequate schooling, children with dyslexia have greater difficulty with reading than with other cognitive abilities, which appear normal. ${ }^{1)}$ They usually experience difficulties with accurate and/or fluent word recognition and exhibit poor spelling and decoding abilities. ${ }^{2)}$ The prevalence rates of dyslexia range from 5 to $10 \%$ in school-age children, ${ }^{3)}$ and $30-50 \%$ have a family history of dyslexia. ${ }^{4,5)}$

Despite their knowledge of orthographical and gram-

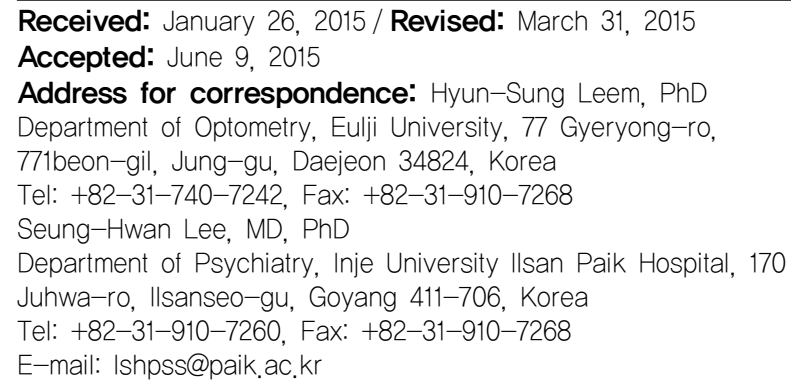


hance the right hemisphere regions, thus supporting reading accuracy. Indeed, activation of the right hemisphere is negatively associated with the reading ability of individuals with dyslexia. ${ }^{17-19)}$

The use of event-related potentials (ERPs) allows the investigation of rapid information processing. Additionally, its high temporal resolution enables the isolation of the early and late information processing stages that occur within $1 \mathrm{~s}$ and may be related to the core psychopathology of individuals with dyslexia. Taroyan et al. ${ }^{20)}$ investigated correlations related to motion perception in dyslexia by recording ERPs in response to random-dot stimuli that combined low contrast. They found that the P3 amplitude was increased in the right hemisphere of both the dyslexic and control groups. Araújo et al. ${ }^{21)}$ conducted an implicit reading task in pre-adolescent children with dyslexia. Significant differences in the early ERP components (P1 and N1) were observed in only the control group when comparing consonant and symbol sequences; however, these differences were not observed in the dyslexic group. They interpreted these data as indicative a lack of visual specialization for letter processing in participants with dyslexia. In addition, Csépe et al. $^{22)}$ revealed that the P100 amplitude was increased in dyslexia adults during the stage of lexical access while they were performing a task. The late component was also increased in dyslexic adults compared with the control group. They interpreted these increased early and late ERP amplitudes as compensatory attentional efforts made by dyslexia adults. Dhar et al. ${ }^{23)}$ used a cue-target processing task, including valid/invalid conditions, and found that adults with dyslexia showed larger N2 amplitudes in the right hemisphere under the valid condition. However, the sample for this experiment included only adult males, and relatively little research using ERPs had been conducted on children with developmental dyslexia.

Our study was designed based on previous research that reported abnormal findings at $\mathrm{P} 100$ and $\mathrm{P} 300$ in children with dyslexia. P100 is a prominent component involved in early sensory processing, and it is also useful for studying the visual attention processing of the parietal-occipital region. Johannes et al. ${ }^{24)}$ found that P100 was influenced by luminance and spatial attention. A later component, P300, which reaches the parietal region in post-sensory processing, is used as a measure of conscious attentional allocation and effortful processing. ${ }^{25)}$ These two ERP components may be useful in investigations of early and late information processing in children with developmental dyslexia. Therefore, we chose these parameters to explore spatial and conscious attention in the occipital and parietal regions.

The present study examined children's ERP responses while performing the Posner spatial cueing task ${ }^{26)}$ in which a spatial cue is followed by a target. This type of target-detection task is considered to be effective for evaluating covert attention during visual processing. We hypothesized that children with dyslexia would exhibit later but relatively accurate responses to stimuli that required processing involving visual attention. We also hypothesized that this type of visual processing would be observed early and late in processing. In addition, we expected increased difficulty and more effortful processing in the right hemisphere than in the left hemisphere during attentional processing.

\section{METHODS}

\section{Participants}

Seventeen children (eight males and nine females) with dyslexia (mean age, 9.13 years; standard deviation [SD], $1.44)$ and 18 healthy control children (10 males and 8 females; mean age, 9.61 years; $\mathrm{SD}, 1.10$ ) participated in this experiment. The children with dyslexia were recruited from patients visiting the Child and Adolescent Psychiatry Clinic of the Inje University Ilsan Paik Hospital (Goyang, Korea) and from an Internet community for children with dyslexia. Healthy controls were recruited from the local community through flyers. Participants with any history of neurological illness other than dyslexia- such as attention-deficit/hyperactivity disorder (ADHD), learning disabilities, or mental retardation - were excluded from this study. All participants with dyslexia had sought help from mental health services and had been formally diagnosed according to Diagnostic and Statistical Manual of Mental Disorders 4th edition, text revision (DSM-IV-TR) ${ }^{27)}$ criteria for reading disorder by board-certified psychiatrists. Intelligence was assessed with the Korean-Wechsler Intelligence Scale for Children IV (K-WISC) ${ }^{28}$. The Korean-ADHD Rating Scale (K-ARS) ${ }^{29)}$ was also administered to rule out the possibility of ADHD.

All participants had normal or corrected-to-normal vision and an intelligence quotient (IQ) of at least 85 . The mean IQ of the control group was 118 (SD, 6.74), and that of the dyslexic group was 113 (SD, 7.44). There was no significant difference in the IQs of the two groups $(p=0.10)$ (Table 1). All participants were right-handed, which was determined by asking the participants which hand they used most for writing and other activities. 
Table 1. Mean age, $1 Q$, K-LDES, and K-ARS in the children with and without dyslexia

\begin{tabular}{lrrc}
\hline \multicolumn{1}{c}{ Variable } & Control $(n=18)$ & Dyslexia $(n=17)$ & p value \\
\hline Age & $9.61(1.10)$ & $9.13(1.44)$ & 0.215 \\
IQ & $118.00(6.74)$ & $113.94(7.44)$ & 0.104 \\
K-LDES (spelling) & $10.28(1.41)$ & $4.71(1.21)$ & 0.000 \\
K-LDES (reading) & $10.06(1.43)$ & $4.47(1.33)$ & 0.000 \\
K-ARS & $4.83(3.87)$ & $7.59(3.39)$ & 0.033 \\
\hline
\end{tabular}

Values are presented as mean (standard deviation).

$1 Q$, intelligence quotient; K-LDES, Korean Learning Disability Evaluation Scale; K-ARS: Korean Attention-Deficit/Hyperactivity Disorder Rating Scale.

Our experimental procedures were approved by the Institutional Review Board of Inje University Ilsan Paik Hospital (IB-3-1212-051). We obtained written informed consent from all participants as well as from their next of kin, caretakers, or guardians on behalf of all of the minors/children enrolled in our study before the experiment began.

\section{Psychological Measures}

All participants were screened using the spelling and reading scores of the Korean Learning Disability Evaluation Scale (K-LDES) ${ }^{30)}$ the Basic Learning Skills Test, the Korean-ADHD Rating Scale (K-ARS), ${ }^{29)}$ and a pediatric neuropsychiatric interview. The K-LDES consists of the bottom measure of the seven following attention, thinking, speaking, reading, writing, spelling, and mathematical calculus. Standard scores range from 0 to 18 points, and individuals with standardized spelling and readings less than 6 points and other scores greater than 7 points were classified as dyslexic. The reading portion of the Basic Learning Skills Test is composed of a total of 50 words. Participants who experienced difficulty reading more than five words were placed in the dyslexic group. The total possible score on K-ARS is 54 points; a score less than 18 points on the portion of the test completed by the person who completed the informed consent form was used to identify children without ADHD.

The following inclusion criteria were used for the developmental dyslexia group: 1) a maximum score of 6 points each on the standardized spelling and reading tests of the K-LDES, ${ }^{31)}$ and 2) a minimum score of 5 on Part I on the Basic Learning Skills Test. ${ }^{32)}$ In addition, all the participants had to score less than 18 points on the K-ARS to indicate that they did not exhibit symptoms of ADHD. ${ }^{33)}$ Intelligence was assessed using the K-WISC. ${ }^{28)}$

\section{Event-related Potential Paradigm}

During the electroencephalography (EEG) session, participants were seated approximately $60 \mathrm{~cm}$ from a

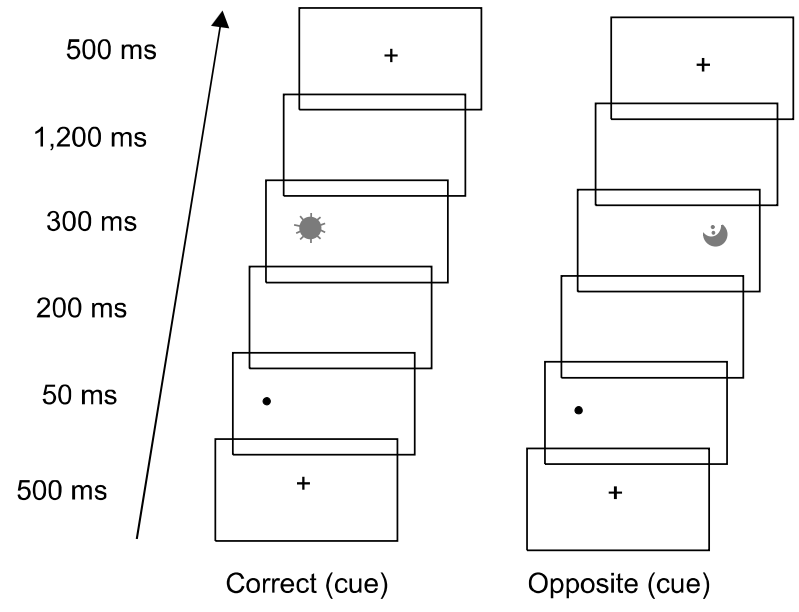

Fig. 1. Sequence and duration of the experiment for the cue target task. The participants had to press the " 1 " on the keyboard when they saw the sun and "2" when the moon was displayed.

computer screen in a sound-attenuated, dimly lit EEG room. Each task trial consisted of the presentation of a central fixation dot for $500 \mathrm{~ms}$, followed by the presentation of a peripheral cue, which appeared either to the left or to the right of the fixation dot, for $50 \mathrm{~ms}$. After a 200-ms interval, a subsequent target object was presented for $300 \mathrm{~ms}$. Following the presentation of the target object, a 1,200-ms interval was inserted before the next trial. The cue either accurately predicted the target location or appeared on the opposite side of the screen (correct: 120 trials [37.5\%] vs. opposite: 120 trials [37.5\%]). The study also included a no-cue condition (no cue: 80 trials, $25 \%$ ) in which the target object was either a picture of a sun or a moon that was adjusted for contrast and luminescent balance using Adobe Photoshop 7.0 (Adobe Systems Inc., San Jose, CA, USA). The cue and target were presented randomly, and the subjects were required to press the appropriate keyboard button (the number " 1 " in response to the sun and the number " 2 " in response to the moon) as soon as possible. Before the experiment, the participants performed a practice session to ensure that they understood the task. Data from the correct-cue condition were analyzed in this study.

We used two measures assess participants' performance with regard to distinguishing between the two possible targets: 1) response accuracy, and 2) reaction time (RT). Mean RT was calculated only for the trials in which correct responses were provided. Any responses before target onset or 1,200 ms following target offset were considered incorrect. Figure 1 shows the sequence and duration of this experimental task. 


\section{Electrophysiological Recording and Analysis}

EEG signals were recorded using NeuroScan SynAmps (Compumedics USA, El Paso, TX, USA) with 64 $\mathrm{Ag}-\mathrm{AgCl}$ electrodes mounted on a Quick Cap. The electrodes were attached according to the extended 10-20 system. ${ }^{34)}$ Reference electrodes were attached to both mastoids, a ground electrode was located on the forehead, the vertical electrooculogram (EOG) was attached above and below the left eye, and the horizontal EOG was attached at the outer canthus of each eye. The sampling rate was set at 1,000 Hz. E-Prime (Psychology Software Tools Inc., Pittsburgh, PA, USA) was used to synchronize the exact stimulus onset with the recorded signal.

The recorded EEG was preprocessed using Scan 4.3 to reduce various artifacts. Gross movement artifacts were removed from the recorded data by visual inspection, and eye blinks were removed using an established mathematical procedure. ${ }^{35)}$ Any signal exceeding $\pm 75 \mu \mathrm{V}$ was regarded as a physiological artifact, and the corresponding epoch was eliminated from the analysis. The raw signal was re-referenced to an average reference, and the data were divided into epochs lasting from $200 \mathrm{~ms}$ to $800 \mathrm{~ms}$. Baseline correction was done by subtracting the mean activity prior to stimulus onset. A band-pass filter $(1-30 \mathrm{~Hz})$ was applied to the epoch. ${ }^{36}$ )

We examined the ERPs generated in response to targets. P100 was examined in the parietal-occipital region and was defined as the most positive peak during the 100-150 $\mathrm{ms}$ interval after target onset. The average for P100 was derived from $\mathrm{P} 5 / \mathrm{P} 7 / \mathrm{PO} 7$ in the left hemisphere and $\mathrm{P} 6 / \mathrm{P} 8 / \mathrm{PO} 8$ in the right hemisphere. $\mathrm{P} 300$ was examined in the parietal region and was defined as the most positive peak in the $300-400 \mathrm{~ms}$ interval after target onset. It was examined in $\mathrm{F} 3 / \mathrm{F} 4, \mathrm{C} 3 / \mathrm{C} 4$, and $\mathrm{P} 3 / \mathrm{P} 4$.

We used the lateral asymmetry index (LAI) to analyze asymmetry. ${ }^{37)}$ The LAI was determined by comparing the corresponding ERP amplitudes for the left and right hemispheres. It was computed by dividing the differences between the two hemispheres by their sum (i.e., $\mathrm{LAI}=\left[P_{\text {left }}-\right.$ $\left.P_{\text {right }}\right] /\left[P_{\text {left }}+P_{\text {right }}\right]$, where $P_{\text {left }}$ and $P_{\text {right }}$ are the relative amplitudes of the corresponding ERP component in the appropriate brain region). The resulting values ranged from 1 (when the right hemisphere showed zero activity) to -1 (when the left hemisphere showed zero activity). An index of zero indicated equivalent activity in both hemispheres. A positive LAI reflects dominant brain activity in the left hemisphere, whereas a negative LAI reflects dominant brain activity in the right hemisphere.

\section{Statistical Analysis}

Our data did not satisfy the requirements of a normal distribution. Mann-Whitney $U$-tests were performed to compare the two groups with regard to age, IQ, and behavioral data. Data on ERP amplitude and latency were analyzed with a repeated-measure ANOVA treating group (dyslexia vs. healthy controls) as the between-subjects factor and laterality (right vs. left) as the within-subject factor. The P300 analysis treated three regions (frontal vs. central vs. parietal) as the within-subjects factor. Post hoc analysis was conducted with Bonferroni's corrected independent $t$-test. Statistical significance was set at $p<0.05$.

PASW Statistics software ver. 18.0 (IBM Co., Armonk, NY, USA) was used for the statistical analysis.

\section{RESULTS}

\section{Behavioral Data}

The dyslexic group showed significantly longer RTs (666.71 [190.72] ms vs. 574.00 [70.74] ms, $p=0.007$ ) compared with the healthy control group in the target-detection task. However, we found no significant differences between the two groups with regard to accuracy $(0.888$ [0.09] vs. 0.919 [0.08], $p=0.338$ ).

\section{Event-related Potential Results}

Table 2 presents the amplitudes and latencies of P100 and P300 in children with dyslexia and healthy controls.

Table 2. Comparison of $\mathrm{P} 100$ and $\mathrm{P} 300$ amplitude and latency between children with and without dyslexia

\begin{tabular}{ccc}
\hline \multicolumn{1}{c}{ Variable } & Control $(\mathrm{n}=18)$ & Dyslexia $(\mathrm{n}=17)$ \\
\hline P100 Amplitude ( $\mu \mathrm{V})$ & & \\
Left (P5, P7, PO7) & $4.80(6.10)$ & $4.20(3.78)$ \\
Right (P6, P8, PO8) & $3.03(3.43)$ & $6.81(5.76)$ \\
P100 Latency (ms) & & \\
Left (P5, P7, PO7) & $119.35(12.08)$ & $130.12(21.78)$ \\
Right (P6, P8, PO8) & $130.66(13.80)$ & $144.39(21.19)$ \\
P300 Amplitude ( $\mu$ V) & & \\
F3 & $-4.20(4.38)$ & $-5.74(3.88)$ \\
F4 & $-2.24(2.75)$ & $-2.30(3.71)$ \\
C3 & $0.49(2.68)$ & $-0.73(4.99)$ \\
C4 & $0.40(4.08)$ & $-0.57(3.06)$ \\
P3 & $3.40(3.82)$ & $5.34(4.75)$ \\
P4 & $3.86(4.06)$ & $6.88(4.78)$ \\
P300 Latency (ms) & & \\
F3 & $381.22(50.19)$ & $380.47(38.94)$ \\
F4 & $378.27(36.80)$ & $370.82(48.91)$ \\
C3 & $353.28(53.41)$ & $341.71(34.97)$ \\
C4 & $359.22(37.23)$ & $365.82(49.70)$ \\
P3 & $332.44(32.77)$ & $338.88(30.79)$ \\
P4 & $344.83(27.29)$ & $359.82(30.15)$ \\
\hline Val & &
\end{tabular}

Values are presented as mean (standard deviation). 
P100

\section{Amplitude}

No main effect for group and laterality was observed. However, we found a significant interaction between laterality and group $(\mathrm{F}[1,33]=9.374, p=0.04]$. Post hoc analysis revealed that the dyslexic group exhibited larger P100 amplitudes compared with the healthy control group in the right hemisphere (6.81 [5.76] $\mu \mathrm{V}$ vs. 3.03 [3.43] $\mu \mathrm{V}$, $p=0.024)$, but no difference in this regard was observed in the left hemisphere (Figs. 2A, 2B, 3A).

\section{Latency}

We found a significant main effect for group (F $[1,33]=6.550, p=0.015)$ and laterality $(\mathrm{F}[1,33]=12.949$, $p=0.001$ ). Post hoc analysis revealed that the dyslexic group showed significantly delayed P100 latencies compared with the healthy control group $(137.25$ [14.13] ms vs. $125.00[14.11] \mathrm{ms}, p=0.015)$. The right hemisphere of the dyslexic group showed a significant delayed P100 latency compared with the left hemisphere (137.52 [15.00] ms vs. $124.73[14.75] \mathrm{ms}, p=0.001)$. There were no sig- nificant group-related interactions with regard to P100 latency.

\section{P300}

Amplitude

Significant main effects were observed for region (F $[2,32]=43.193, p<0.001)$ and laterality $(\mathrm{F}[1,33]=5.776$, $p=0.022$ ). Post hoc analysis revealed that the parietal electrode exhibited larger P300 amplitudes compared with those of the central and frontal electrodes $(4.87[3.15] \mu \mathrm{V}$ vs. $-0.10[2.05] \mu \mathrm{V}$ vs. $-3.62[2.85] \mu \mathrm{V})$. The right hemisphere of the dyslexic group showed significant larger P300 amplitudes compared with the left hemisphere $(1.00[1.45] \mu \mathrm{V}$ vs. -0.23 [1.50] $\mu \mathrm{V}, p=0.022)$ (Figs. $4 \mathrm{~A}, 4 \mathrm{~B}, 3 \mathrm{~B}$; lower part). There were no significant group-related interactions with regard to $\mathrm{P} 300$ amplitudes.

\section{Latency}

The data revealed significant main effects for region (F $[2,32]=8.042, p=0.001)$. Post hoc analysis revealed that data from the parietal electrode reflected shorter P300

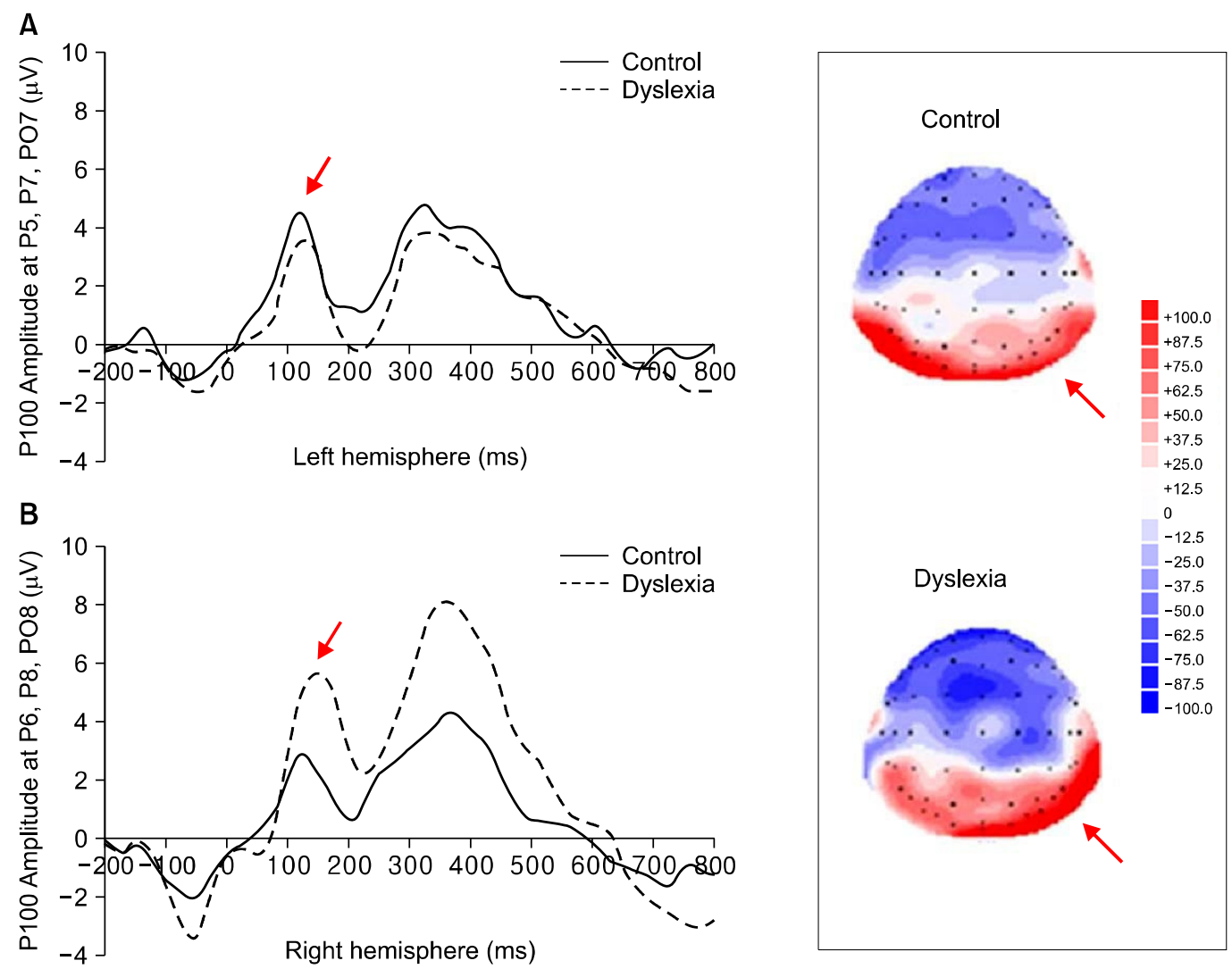

Fig. 2. The left figures are the grand average waves of P100. Two groups are represented by solid and dotted lines. (A) Left hemisphere; P5, P7, PO7. (B) Right hemisphere; P6, P8, PO8. The right figure shows the topography at P100, which is displayed with colors. 

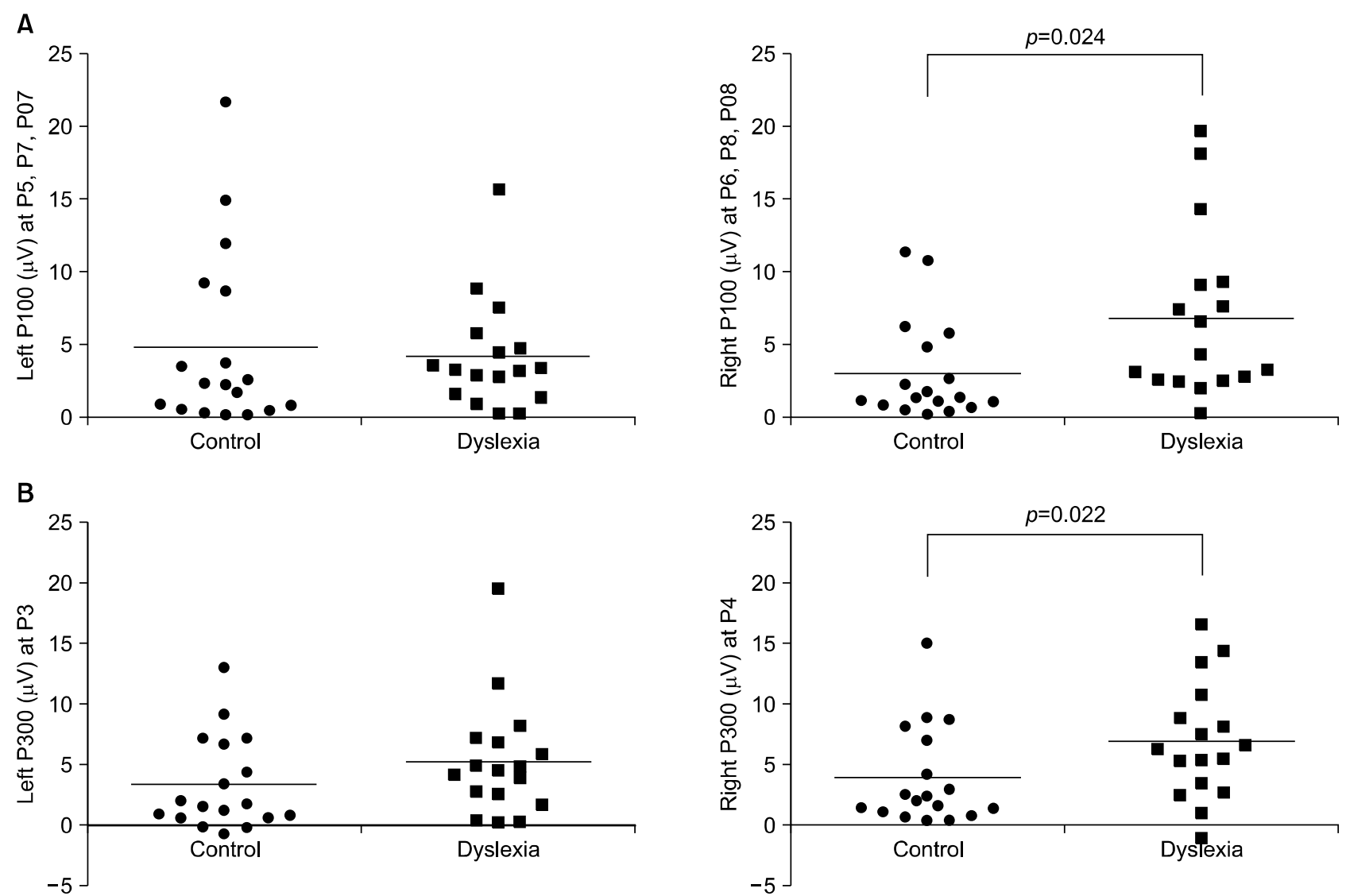

Fig. 3. Scattergrams. (A) The upper figures are the scattergrams of averaged $P 100$ amplitudes (P5, P7, PO7 vs. P6, P8, PO8) at each hemisphere of two groups. (B) The lower figures are the scattergrams of P300 amplitudes (P3 vs. P4) at each hemisphere of two groups.

latencies compared with those from the central and frontal electrodes (343.99 [22.4] ms vs. 355.00 [30.1] ms vs. 377.69 [33.35] ms). There were no significant group-related interactions in P300 latency.

\section{Lateral Asymmetry Index and Correlations}

Table 3 presents the LAIs for P100 and P300 in children with dyslexia and healthy controls. We found a significant difference between the two groups with regard to the LAI (0.083 [0.33] vs. -0.206 [0.35], $p=0.029)$, which indicates the dominance of the right hemisphere for P100 in children with dyslexia. However, no significant differences were observed for P300 ( -0.108 [0.44] vs. -0.155 [0.49], $p=0.232$ ). The accuracy of the dyslexic group in the target-detection task was significantly correlated with their LAI for P100 $(r=-0.513, p=0.035)$, but there were no significant correlations related to their RTs $(r=0.260$, $p=0.314$ ) (Fig. 5). No significant correlations with regard to the LAIs of healthy controls were observed.

\section{DISCUSSION}

This study investigated the ERP responses and hemispheric differences between children with developmental dyslexia and children without dyslexia during a visually cued target-detection task. Our study revealed that children with dyslexia had slower RTs compared with controls. Children with dyslexia show delayed P100 latencies and increased P100 amplitudes in the right hemisphere compared with children without dyslexia. The P300 amplitudes of the right hemisphere were significantly increased compared with those of the left hemisphere in the dyslexic group. The LAIs for P100 reflected the significant dominance of the right hemisphere in children with dyslexia and were significantly negatively correlated their accuracy in the target-detection task.

Our study revealed that children with dyslexia exhibit slower RTs, indicating that children with this disorder require more time than children without it to shift their attention to the target. Numerous previous studies have found that children with dyslexia demonstrate sluggish and slow 

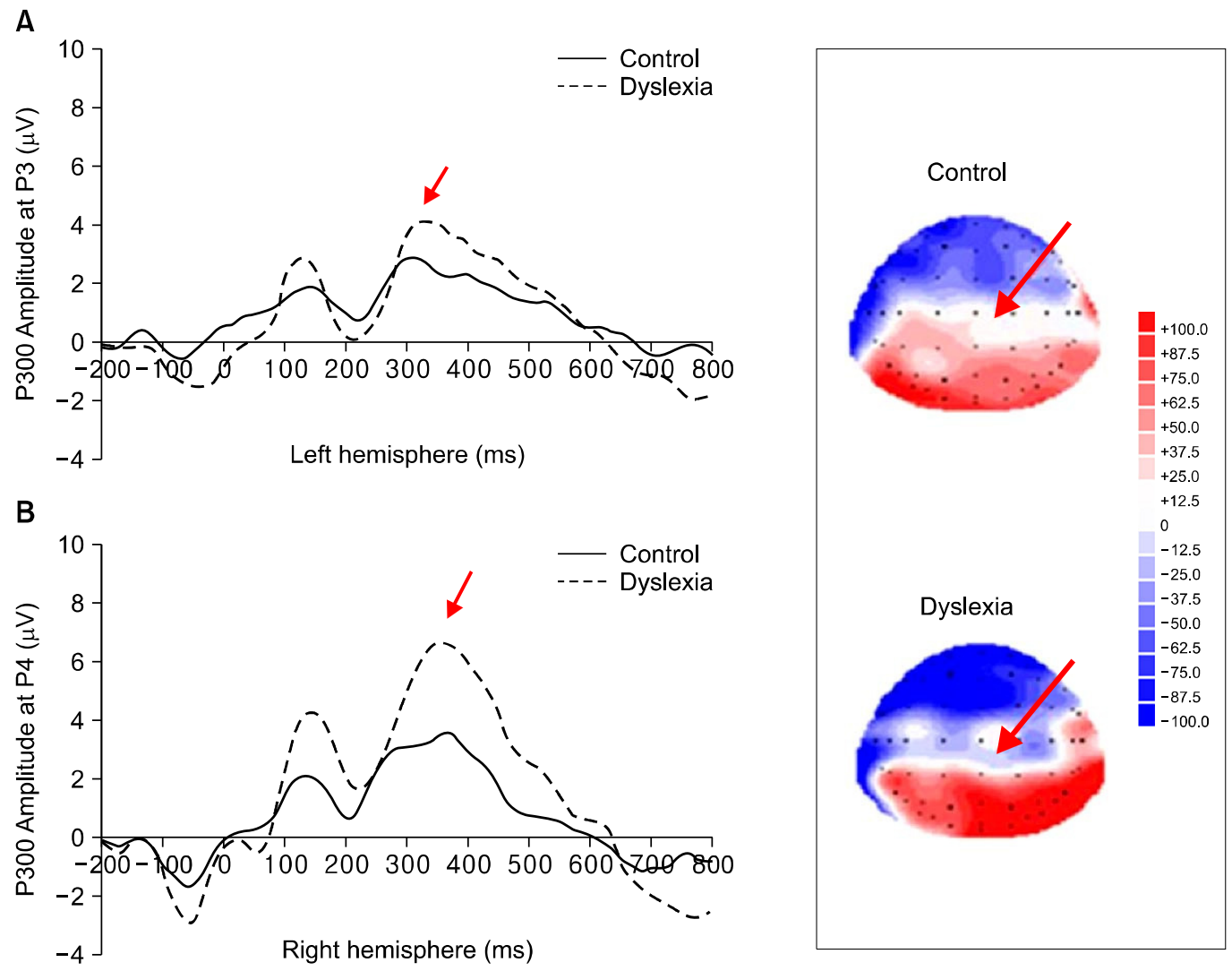

Fig. 4. The left figure are the P300 waves at P3 and P4. The two groups are represented by solid and dotted lines. (A) Left hemisphere; P3. (B) Right hemisphere; P4. The right figure shows the topography at P300, which is displayed with colors.

Table 3. Lateral asymmetry index at P100 and P300

\begin{tabular}{rrrc}
\hline & Control $(n=18)$ & Dyslexia $(n=17)$ & $p$ value \\
\hline P100 & $0.083(0.33)$ & $-0.206(0.35)$ & 0.029 \\
P300 & $-0.108(0.44)$ & $-0.155(0.49)$ & 0.232 \\
\hline
\end{tabular}

Values are presented as mean (standard deviation). $\mathrm{P} 100=((\mathrm{P} 5+\mathrm{P} 7+\mathrm{PO} 7)-(\mathrm{P} 6+\mathrm{P} 8+\mathrm{PO} 8)) /((\mathrm{P} 5+\mathrm{P} 7+\mathrm{PO} 7)+(\mathrm{P} 6+\mathrm{P} 8+\mathrm{PO} 8))$. $\mathrm{P} 300=(\mathrm{P} 3-\mathrm{P} 4) /(\mathrm{P} 3+\mathrm{P} 4)$.

attentional shifts when performing a visual task. ${ }^{38,39)}$ Buchholz and Aimola Davies ${ }^{40)}$ also reported that adults with dyslexia are generally slower to detect targets. These delayed RTs were interpreted as evidence that dyslexia causes slow information processing. ${ }^{41)}$

However, we found no significant difference between the accuracy with which the two groups detected targets. This finding indicates that children with dyslexia may develop compensatory reading systems to read and write accurately. ${ }^{42)}$ Moreover, such compensatory systems may play a prominent role in the ability of many people with dyslexia to read and accurately detect targets given that the academic and attentional performance of such individuals are at least normal.
Compared with controls, children with dyslexia revealed significantly delayed P100 latencies in the right parietal-occipital hemisphere; however, this kind of slowness was not observed in the left hemisphere. Stefanics et $a{ }^{43)}$ found slower P100 responses in children with dyslexia in an auditory sensory processing task involving the use of an oddball condition. Many previous studies have reported delayed ERP latencies (N100, P150, P200) when individuals with dyslexia were in the early informationprocessing stages of the syntactic functions of words, ${ }^{44)}$ orthographic/phonological modalities, ${ }^{45)}$ and visual information. ${ }^{46)}$ Csépe et al. ${ }^{22)}$ found a large increase in P100 amplitudes in children with dyslexia and argued that these data reflected the effort required from these children during the stage of lexical access. Our results are consistent with these previous findings.

These results suggest that children with dyslexia may use a different strategy, involving right hemispheric neural pathways, for early visual processing compared with children without dyslexia. Additionally, P300 amplitudes were significantly increased in the right hemisphere compared with left hemisphere in the dyslexia group. P300 has 

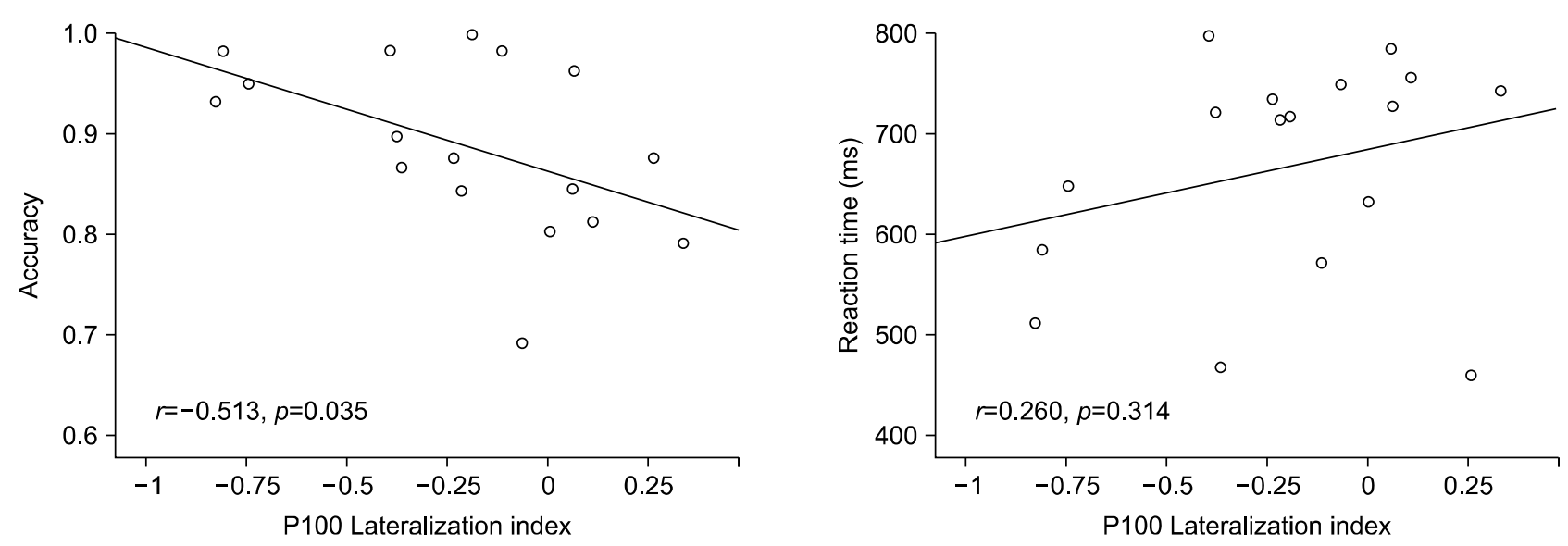

Fig. 5. Pearson correlations between the lateral asymmetry index of P100 amplitude with accuracy/reaction times during a cue target task performed by children with dyslexia.

been regarded as involved in late-phase conscious attentional processing, ${ }^{47)}$ effortful processing, ${ }^{25)}$ and discriminating differences among several different stimuli. ${ }^{23)}$ Although we did not find group differences in P300 amplitudes, the results of our hemispheric analysis of P300 responses are consistent with previous reports showing the dominance of the right hemisphere of P300 in visuospatial attention $^{48)}$ and auditory oddball tasks. ${ }^{49)}$ Taroyan et al. ${ }^{20)}$ also reported larger right hemisphere P300 amplitudes in participants with dyslexia during a visual attention task. Breznitz and Leikin ${ }^{41)}$ suggested that a significant increase in P300 amplitude seems to improve sentence comprehension when processing words with different syntactic functions. However, several studies have shown both smaller $^{50,51)}$ and comparable P300 amplitudes $^{52)}$ in individuals with dyslexia compared with controls.

Data on the LAI for P100 amplitude reflect the significant dominance of the right hemisphere in children with dyslexia. Moreover, this hemispheric dominance was significantly correlated with the accuracy with which children with dyslexia performed the target-detection task, whereas there was no significant correlation in this regard among healthy controls. Previous studies have repeatedly reported right-hemispheric dominance in individuals with dyslexia. Byring et al. ${ }^{53)}$ found that an EEG correlation index and the intermittent simultaneous activation of the cortical regions involved in a cognitive task ${ }^{54)}$ are high within the right hemisphere of children with reading and writing impairments. Vlachos et al. ${ }^{19)}$ reported that significantly more students with dyslexia than their friends without dyslexia displayed a preference for a right hemispheric thinking style. Chiarello et al. ${ }^{17)}$ suggested that anatomical variations in the right hemisphere may be im- portant contributors to learning to read. Sarkari et al. ${ }^{18)}$ reported an increased right temporo-parietal area in children with dyslexia during a pseudo-word reading task. This dominance of the right hemisphere may be explained by a compensatory mechanism that compensates for the deficits in left hemisphere that are common in individuals with dyslexia. $^{42)}$

This study had the following limitations: 1) the number of participants was relatively small; 2) the correct and opposite conditions were not separately analyzed in the ERP paradigm from the beginning; 3) we did not consider other factors, such as scalp thickness or bone density; and 4) we did not use structured diagnostic and differential diagnostic measures for dyslexia (reading disorder).

In conclusion, our results showed that children with dyslexia provided slower but accurate responses in a target-detection task. Analysis of P100 and P300 amplitudes showed that children with dyslexia predominantly use the right hemisphere when concentrating on a target-detection task. The dominance of the right hemisphere observed in children with dyslexia may reflect an ancillary mechanism that compensates for the poor reading function of the left regions.

\section{Acknowledgments}

This research was supported by the Basic Science Research Program through the National Research Foundation of Korea (NRF), which is funded by the Ministry of Education, Science and Technology (No. 2012R1A1A2043992). The authors wish to thank JC.

\section{REFERENCES}

1. Shaywitz SE, Shaywitz BA. Dyslexia (specific reading 
disability). Biol Psychiatry 2005;57:1301-1309.

2. Lyon GR, Shaywitz SE, Shaywitz BA. A definition of dyslexia. Ann Dyslexia 2003;53:1-14.

3. Habib M. The neurological basis of developmental dyslexia: an overview and working hypothesis. Brain 2000;123 Pt 12:2373-2399.

4. Pennington BF, Lefly DL. Early reading development in children at family risk for dyslexia. Child Dev 2001;72: 16-833.

5. Gilger JW, Pennington BF, Defries JC. Risk for reading disability as a function of parental history in three family studies. Read Writ 1991;3:205-217.

6. Willows D. Visual processing in learning disabilties. In: Wong BYL, editor. Learning about learning disabilities. San Diego, CA:Academic Press; 1998.

7. Gough PB, Ehri L, Treiman R. Reading acquisition. Hillsdale, NJ:Lawrence Erlbaum Associates; 1992.

8. Bosman AM, de Groot AM. Phonologic mediation is fundamental to reading: evidence from beginning readers. $Q J$ Exp Psychol A 1996;49:715-744.

9. Casco C, Tressoldi PE, Dellantonio A. Visual selective attention and reading efficiency are related in children. Cortex 1998;34:531-546.

10. Hari R, Renvall H. Impaired processing of rapid stimulus sequences in dyslexia. Trends Cogn Sci 2001;5:525-532.

11. Facoetti A, Turatto M, Lorusso ML, Mascetti GG. Orienting of visual attention in dyslexia: evidence for asymmetric hemispheric control of attention. Exp Brain Res 2001;138: 6-53.

12. Iles J, Walsh V, Richardson A. Visual search performance in dyslexia. Dyslexia 2000;6:163-177.

13. Facoetti A, Paganoni P, Turatto M, Marzola V, Mascetti GG. Visual-spatial attention in developmental dyslexia. Cortex 2000;36:109-123.

14. Rayner K, Murphy L, Henderson J, Pollatsek A. Selective attentional dyslexia. Cogn Neuropsychol 1989;6:357-378.

15. Geiger G, Lettvin JY. Peripheral vision in persons with dyslexia. $N$ Engl J Med 1987;316:1238-1243.

16. Zoubrinetzky R, Bielle F, Valdois S. New insights on developmental dyslexia subtypes: heterogeneity of mixed reading profiles. PLoS One 2014;9:e99337.

17. Chiarello C, Lombardino LJ, Kacinik NA, Otto R, Leonard CM. Neuroanatomical and behavioral asymmetry in an adult compensated dyslexic. Brain Lang 2006;98:169-181.

18. Sarkari S, Simos PG, Fletcher JM, Castillo EM, Breier JI, Papanicolaou AC. Contributions of magnetic source imaging to the understanding of dyslexia. Semin Pediatr Neurol 2002;9:229-238.

19. Vlachos F, Andreou E, Delliou A. Brain hemisphericity and developmental dyslexia. Res Dev Disabil 2013;34:1536-1540.

20. Taroyan NA, Nicolson RI, Buckley D. Neurophysiological and behavioural correlates of coherent motion perception in dyslexia. Dyslexia 2011;17:282-289.

21. Araújo S, Bramão I, Faísca L, Petersson KM, Reis A. Electrophysiological correlates of impaired reading in dyslexic pre-adolescent children. Brain Cogn 2012;79:79-88.

22. Csépe V, Szücs D, Honbolygó F. Number-word reading as challenging task in dyslexia? An ERP study. Int $J$ Psychophysiol 2003;51:69-83.

23. Dhar M, Been PH, Minderaa RB, Althaus M. Distinct information processing characteristics in dyslexia and ADHD during a covert orienting task: an event-related potential study. Clin Neurophysiol 2008;119:2011-2025.

24. Johannes S, Münte TF, Heinze HJ, Mangun GR. Luminance and spatial attention effects on early visual processing.
Brain Res Cogn Brain Res 1995;2:189-205.

25. Kok A. On the utility of $P 3$ amplitude as a measure of processing capacity. Psychophysiology 2001;38:557-577.

26. Posner MI. Orienting of attention. $Q J$ Exp Psychol 1980;32:3-25.

27. Association AP. Diagnostic and statistical manual of mental disorders, text revision (DSM-IV-TR). Washington, DC: American Psychiatric Association;2000.

28. Wechsler D. WISC-IV. Wechsler intelligence scale for children: technical and interpretative manual. 4th ed. London: Pearson;2003.

29. Kim JW, Park KH, Choi MJ. Screening for attention deficit/hyperactivity disorder in community mental health services for children. $J$ Korean Neuropsychiatr Assoc 2004;43:200-208.

30. Shin MS, Hong KE, Kim ZS, Cho SC. A standardization study of the Korean version of learning disability evaluation scale. J Korean Neuropsychiatr Assoc 1998;37:1233-1245.

31. Song JY, Shin MS. Differencies between reading disabilities and ADHD on K-LDES. Korean J Clin Psychol 1998;17: 185-196.

32. Park KS, Yoon JY, Park HJ. The basic academic skills sample. Seoul:Korean Educational Development Institute;1989.

33. Yang YH, Kim JW, Kim YN, Cho SC, Kim BN. Screening for attention deficit/hyperactivity disorder for children in Seoul. J Korean Neuropsychiatr Assoc 2008;47:292-298.

34. Homan RW, Herman J, Purdy P. Cerebral location of international 10-20 system electrode placement. Electroencephalogr Clin Neurophysiol 1987;66:376-382.

35. Semlitsch HV, Anderer P, Schuster P, Presslich O. A solution for reliable and valid reduction of ocular artifacts, applied to the P300 ERP. Psychophysiology 1986;23:695-703.

36. Bae KY, Kim DW, Im CH, Lee SH. Source imaging of P300 auditory evoked potentials and clinical correlations in patients with posttraumatic stress disorder. Prog Neuropsychopharmacol Biol Psychiatry 2011;35:1908-1917.

37. Lee MS, Lee SH, Moon EO, Moon YJ, Kim S, Kim SH, et al. Neuropsychological correlates of the P300 in patients with Alzheimer's disease. Prog Neuropsychopharmacol Biol Psychiatry 2013;40:62-69.

38. Dykman RA, Ackerman PT, Holcomb PJ, Boudreau AY. Physiological manifestations of learning disability. J Learn Disabil 1983;16:46-53.

39. Wijers AA, Been PH, Romkes KS. Dyslexics show a deviant lateralization of attentional control: a brain potential study. Neurosci Lett 2005;374:87-91.

40. Buchholz J, Aimola Davies A. Adults with dyslexia demonstrate space-based and object-based covert attention deficits: shifting attention to the periphery and shifting attention between objects in the left visual field. Brain Cogn 2005;57: 30-34.

41. Breznitz Z, Leikin M. Effects of accelerated reading rate on processing words' syntactic functions by normal and dyslexic readers: event related potentials evidence. J Genet Psychol 2001;162:276-296.

42. Shaywitz S. Overcoming dyslexia: a new and complete science-based program for reading problems at any level. New York:Knopf;2003.

43. Stefanics G, Fosker T, Huss M, Mead N, Szucs D, Goswami U. Auditory sensory deficits in developmental dyslexia: a longitudinal ERP study. Neuroimage 2011;57:723-732.

44. Leikin M. Processing syntactic functions of words in normal and dyslexic readers. J Psycholinguist Res 2002;31:145-163.

45. Breznitz Z, Misra M. Speed of processing of the visualorthographic and auditory-phonological systems in adult 
dyslexics: the contribution of "asynchrony" to word recognition deficits. Brain Lang 2003;85:486-502.

46. Regtvoort AG, van Leeuwen TH, Stoel RD, van der Leij A. Efficiency of visual information processing in children at-risk for dyslexia: habituation of single-trial ERPS. Brain Lang 2006;98:319-331.

47. Donchin E, Coles MGH. Is the P300 component a manifestation of context updating? Behav Brain Sci 1988; 11:357-374

48. Nobre AC, Sebestyen GN, Miniussi C. The dynamics of shifting visuospatial attention revealed by event-related potentials. Neuropsychologia 2000;38:964-974.

49. Alexander JE, Bauer LO, Kuperman S, Morzorati S, O'Connor SJ, Rohrbaugh J, et al. Hemispheric differences for P300 amplitude from an auditory oddball task. Int $J$ Psychophysiol 1996;21:189-196.

50. Duncan CC, Rumsey JM, Wilkniss SM, Denckla MB,
Hamburger SD, Odou-Potkin M. Developmental dyslexia and attention dysfunction in adults: brain potential indices of information processing. Psychophysiology 1994;31:386-401.

51. Taylor MJ. The role of event-related potentials in the study of normal and abnormal cognitive development. In: Boller $F$, Grafman J, editors. Handbook of neuropsychology, vol. 10. Amsterdam:Elsevier; 1995.

52. Stelmack RM, Saxe BJ, Noldy-Cullum N, Campbell KB, Armitage R. Recognition memory for words and eventrelated potentials: a comparison of normal and disabled readers. J Clin Exp Neuropsychol 1988;10:185-200.

53. Byring RF, Haapasalo S, Salmi T. Adolescents with learning disorders have atypical EEG correlation indices. II. Correlation indices during reading. Clin Neurophysiol 2004; 115:2584-2592.

54. Byring RF. EEG correlation topography in poor spellers. Electroencephalogr Clin Neurophysiol 1986;63:1-9. 